\title{
The roles of rice microRNAs in rice- Magnaporthe oryzae interaction
}

\author{
Yan Li', John Martin Jerome Jeyakumar ${ }^{1}$, Qin Feng ${ }^{1}$, Zhi-Xue Zhao ${ }^{1}$, Jing Fan', \\ Muhammad Ibrahim Khaskheli ${ }^{2,3}$ and Wen-Ming Wang ${ }^{1 *}$
}

\begin{abstract}
MicroRNAs (miRNAs) are a class of small (20-24 nucleotides (nt) long) non-coding RNAs. One mature miRNA can be transcribed from one or more gene loci known as miRNA genes (MIRs). The transcript of a MIR forms a stem-loop structure that is processed into a 20-24-nt miRNA-5p/-3p duplex by RNase III family endoribonucleases such as Dicer-like1 (DCL1). In turn, the overhang ends of the duplex are methylated by HUA ENHANCER 1 (HEN1), generating stabilized mature miRNAs. The mature miRNAs are loaded onto ARGONAUTE (AGO) proteins, forming a miRNAinduced gene silencing complex (miRISC). Then, the miRISC binds to target sites with sequences complementary to the miRNAs, leading to either cleavage or translational inhibition of the target mRNAs, or methylation of the target sequences, resulting in post-transcriptional and transcriptional gene silencing, respectively. In the past decade, more than 700 miRNAs have been identified in rice, a subset of which have been found to be responsive to the rice blast fungus, Magnaporthe oryzae, or its elicitors. Moreover, members of 10 miRNA families have been found to positively or negatively regulate rice defense against $M$. oryzae, namely miR160, miR164, miR166, miR167, miR169, miR319, miR396, miR398, miR444 and miR7695. This review summarizes the identification and functional characterization of the miRNAs, which respond to M. oryzae or its elicitors and describes the current understanding of the complicated but wellorganized network in the context of rice-M. oryzae interaction.
\end{abstract}

Keywords: Dicer-like 1, MicroRNA, miR164, miR167, miR169, miR319, miR396, miR398, miR7695

\section{Background}

Rice is a staple food for half of the world population and thus is one of the most important grains to secure global food supply. Rice blast is one of the most devastating diseases of rice, and hence threatens food security. Exploitation of blast-resistant cultivars is rated to be the most appropriate strategy to control this disease. Rice mounts an innate immune system consisting of two perception layers to defend itself against invasion by the blast fungus, Magnaporthe oryzae. The first layer of immunity, known as pathogen-associated molecular pattern- (PAMP-) triggered immunity (PTI), is activated following recognition of the fungal pattern molecule chitin, by four lysin motif(LysM-) containing protein (LYP) receptors, i.e., chitin elicitor binding protein $(\mathrm{CEBiP})$, chitin elicitor receptor

\footnotetext{
* Correspondence: j316wenmingwang@sicau.edu.cn

${ }^{1}$ Rice Research Institute and Research Center for Crop Disease and Insect

Pests, Sichuan Agricultural University at Wenjiang, 211 Huimin Road,

Wenjiang District, Chengdu 611130, China

Full list of author information is available at the end of the article
}

kinase (CERK1), LYP4 and LYP6 (Shimizu et al. 2010; Liu et al. 2012). Virulent rice blast strains employ effectors, such as secreted LysM protein1 (Slp1), or avirulence genes, such as AVR-Pizt, to subvert PTI, leading to effector-triggered susceptibility (Mentlak et al. 2012; Park et al. 2016; Wang et al. 2017). In turn, rice resistance $(R)$ genes recognize avirulence effectors to activate the second layer of immunity, i.e., effector-triggered immunity (ETI), to contain the M. oryzae infection (Bialas et al. 2018). To date, more than $30 R$ genes, most of which encode nucleotide-binding site leucine-rich repeat (NLR) family receptors, have been functionally characterized from rice, and nine associated cognate avirulence effectors from $M$. oryzae have been identified (Wang et al. 2017; Xie et al. 2019). Some of these $R$ genes have been widely exploited in rice production and have made major contributions to the control of rice blast. However, activation of immunity often results in yield penalties in rice, known as fitness costs. Some recent reports have demonstrated that both PTI and ETI can be deliberately regulated by immunity-

C The Author(s). 2019 Open Access This article is distributed under the terms of the Creative Commons Attribution 4.0 International License (http://creativecommons.org/licenses/by/4.0/), which permits unrestricted use, distribution, and reproduction in any medium, provided you give appropriate credit to the original author(s) and the source, provide a link to the Creative Commons license, and indicate if changes were made. The Creative Commons Public Domain Dedication waiver (http://creativecommons.org/publicdomain/zero/1.0/) applies to the data made available in this article, unless otherwise stated. 
associated regulators that could reduce or abolish the fitness cost (Deng et al. 2017; Wang et al. 2018b; Chandran et al. 2019). MicroRNAs (miRNAs) are among such regulators capable of fine-tuning growth, development and immunity.

miRNAs are a class of small non-coding RNAs transcribed from miRNA genes (MIRs). To date, more than 500 $M I R$ genes have been identified in the rice genome, generating more than 700 mature miRNAs (Tang and Chu 2017). MIR genes are transcribed into primary miRNAs (pri-miRNAs) that are spliced to form stem-loop precursor miRNAs (pre-miRNAs). The pre-miRNAs are processed into miRNA-5p/miRNA-3p (previously miRNA/miRNA") duplex by Dicer-like1s (DCL1s) or DCL3 (Rogers and Chen 2013). While DCL3 produces mainly 24-nt miRNAs, DCL1s produce 21-nt miRNAs (Liu et al. 2005; Wu et al. 2010; Wei et al. 2014). The overhang ends of the miRNA5p/miRNA-3p duplex are methylated by HUA ENHANCER 1 (HEN1) to stabilize the duplex (Abe et al. 2010). Then, the 21-nt and 24-nt miRNAs are loaded to ARGONAUTE $1 \mathrm{~s}$ (AGO1s) and AGO4, respectively, forming miRNA-induced gene silencing complex (miRISC) (Seo et al. 2013). Whereas the miRNA-AGO1 miRISC mediates sequence-complementary transcript cleavage or translational inhibition, the miRNA-AGO4 miRISC mediates target site methylation (Wu et al. 2009; Wu et al. 2010). Thus, miRNAs negatively regulate the expression of their target genes at either the transcriptional or post-transcriptional level (Fig. 1).

Evidence from Arabidopsis has demonstrated the finetuning roles of miRNAs in both PTI and ETI. The conserved flagellin peptide flg22, a PAMP molecule, induces the expression of a subset of miRNAs, including miR160 and miR393 (Li et al. 2010a). miR393 is the first miRNA to be identified to act in both PTI and ETI against Pseudomonas syringe pv. tomato (Pst) DC3000 via targeting of the auxin receptor genes TIR1, AFB2 and $A F B 3$ to block auxin signaling. On the one hand, the expression of miR393 is induced by flg22 to promote basal defense against bacterial strains (Navarro et al. 2006). The expression of miR393 can be suppressed by AvrPto and AvrPtoB, two bacterial effectors that can subvert PTI (Navarro et al. 2008). On the other hand, both miR393-5p and miR393-3p can be up-regulated during

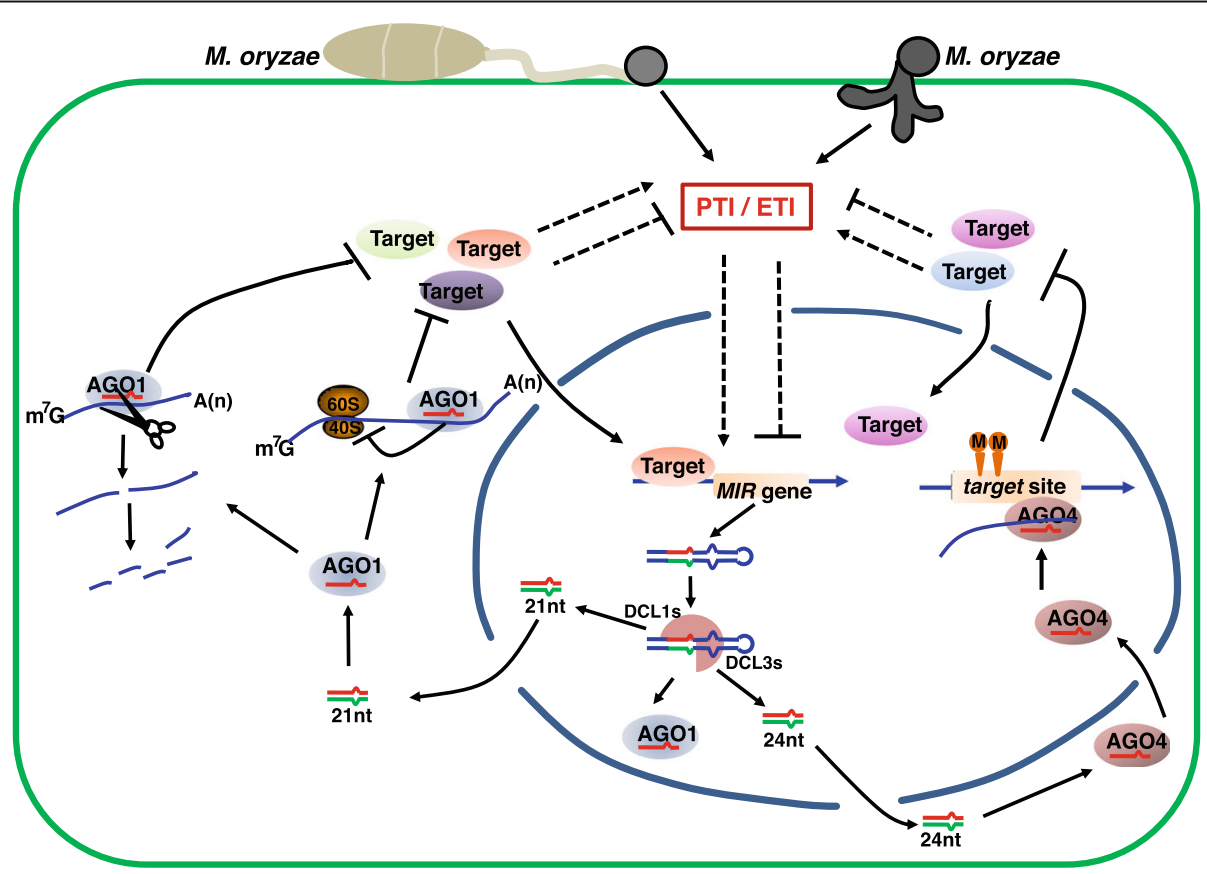

Fig. 1 Hypothetical microRNA-mediated feedback regulation of rice immunity against Magnaporthe oryzae. Infection by M. oryzae activates pathogenassociated molecular pattern- (PAMP-) triggered immunity (PTI) and/ or effector-triggered immunity (ETI). During PTI/ETI, a subset of miRNA genes (MIRs) can be induced or suppressed, depending on their roles as positive or negative regulators, respectively. The MIRs are transcribed and processed by the RNase III family endoribonucleases such as Dicer-like1s (DCL1s) and DCL3s. While DCL3s mainly produce 24-nt (nucleotide) miRNAs, DCL1s produce 21-nt miRNAs. After modification by a series of regulators, the mature single strand 21-nt and 24-nt miRNAs are loaded into ARGONAUTE1 (AGO1) and ARGONAUTE 4 (AGO4), respectively, forming miRNA-induced gene silence complex (miRISCS). The miRISC could then hypothetically mount feedback regulation on PTI/ETI at several layers. First, the 24-nt-AGO4 miRISC re-enters the nucleus to mediate methylation of the DNA sequences complementary to the 24-nt miRNAs, resulting in transcriptional gene silencing of target genes that act in the regulation of PTI/ETI. Second, the 21-nt-AGO1 miRISC mediates the cleavage or translational inhibition of the target mRNAs, leading to post-transcriptional gene silencing of target genes that act in the regulation of PTI/ETI. Third, some target genes of miRNAs are transcription factors that can regulate the transcription of MIR genes and/or genes acting in the regulation of PTI/ETI, forming feedback regulatory networks 
ETI as a result of the recognition of the avirulent effector AvrRpt2 by the $\mathrm{R}$ receptor RPS2 (Zhang et al. 2011). Whereas miR393-5p is loaded onto AGO1, miR393-3p is loaded onto AGO2 and mediates suppression of a SNARE (soluble $N$-ethylmaleimide-sensitive factor attachment protein receptor) gene, $M E M B 12$, to promote immunity against this bacterial pathogen (Zhang et al. 2011). Therefore, a miRNA may be involved in both PTI and ETI, and thus be involved in host-pathogen "arms race". In addition, several miRNAs directly target resistance genes encoding NLR family proteins that mediate ETI, such as miR2109 in Medicago (Zhai et al. 2011), miR482 and miR2118 in tomato (Shivaprasad et al. 2012) and miR472 in Arabidopsis (Boccara et al. 2014). miR472 targets NLRs, such as RPS5 that recognizes the effector AvrPphB to activate ETI against $P$. syringae in Arabidopsis (Boccara et al. 2014). In rice, miRNAs are well-documented in finetuning many biological processes to control many important agronomic traits, such as tiller development, flowering time, panicle establishment, grain formation and yield production, as well as responses to biotic and abiotic stresses (Wu et al. 2015; Bakhshi et al. 2016; Tang and Chu 2017; Wu et al. 2017; Tripathi et al. 2018; Yao et al. 2019). Increasing numbers of reports have shown that miRNAs also play important roles in rice-blast fungus interactions. Because detailed reviews on the biogenesis and functions of miRNAs have been published (Xie et al. 2015; Baldrich and San 2016; Huang et al. 2016b; Tang and Chu 2017; Song et al. 2019), we focus in the current review on rice miRNAs that are responsive to $M$. oryzae or its elicitors, and summarize our understanding of their roles in rice- $M$. oryzae interaction.

\section{Core components of the miRNA signaling pathway involved in rice- $M$. oryzae interaction}

DCLs and AGOs are two key groups of core component proteins in the miRNA signaling pathway, encoded separately by distinct groups of genes. The rice genome contains eight $D C L$ and $19 A G O$ genes (Kapoor et al. 2008). Evidence is emerging that both DCLs and AGOs play important regulatory roles in rice immunity against $M$. oryzae. For example, AGO4 has been demonstrated to regulate the expression of the blast-susceptibility NLR receptor gene PigmS, which, in turn, balances the tradeoff between yield formation and activation of immunity against $M$. oryzae mediated by the broad-spectrum blast resistance gene PigmR (Deng et al. 2017), indicating that AGO4 positively regulates PigmR-mediated ETI. However, the roles of the other AGOs in rice immunity against $M$. oryzae remain to be elusive. Among the eight DCLs, OsDCL1a, OsDCL1b, OsDCL2a/b, OsDCL3a and OsDCL4 have been shown to be responsive to $M$. oryzae
(Zhang et al. 2015; Salvador-Guirao et al. 2019), implying an involvement in rice- $M$. oryzae interaction.

To date, of the eight rice $D C L$ genes, only OsDCL1a has been deeply investigated, being found to negatively regulate PTI against $M$. oryzae. Knock-down of OsDCL1a led to enhanced resistance to the blast disease (Zhang et al. 2015). This resistant phenotype could be due to constitutive activation of defense-related genes, such as 13 Pathogenesis-related genes (PRs) and the PTI-related genes OsKS4 and OsNAC4, as well as higher and faster production of $\mathrm{H}_{2} \mathrm{O}_{2}$ in the knock-down mutant than in the control (Zhang et al. 2015). By contrast, up-regulation of OsDCL1a led to increased susceptibility to both M. oryzae and the bakanae disease fungus Fusarium fujikuroi in an activation-tagged mutant (SalvadorGuirao et al. 2019). Moreover, such activation mutation also resulted in reprogramming of both the transcriptome and the miRNAome, leading to suppression of defense-related genes, phytoalexin biosynthesis and ROS detoxification (Salvador-Guirao et al. 2019). These reports indicate that the functions of $D C L 1$ in rice differ from those in Arabidopsis, in which DCL1 is required for PTI against bacterial pathogens (Navarro et al. 2008).

Consistent with the function of OsDCL1 in the biogenesis of miRNAs, the up-regulation of this gene remarkably influenced the expression of more than 90 miRNAs from 61 miRNA families, including up-regulation and down-regulation of a subset of miRNAs, which, in turn, resulted in altered expression of 216 genes in the categories of "biotic stress," "signaling," and "metabolism" (Salvador-Guirao et al. 2019).

Therefore, OsDCL1s seem to act as a key node in the miRNA signaling pathway, mediating crosstalk between the miRNA network and rice- $M$. oryzae interaction (Fig. 1).

\section{Identification of rice miRNAs responsive to $M$. oryzae or its elicitors}

To investigate the roles of miRNAs in rice- $M$. oryzae interaction, it is vital to first identify those miRNAs that are responsive to $M$. oryzae or its elicitors. A number of research groups have independently identified such miRNAs, as a result of using one of three methods described below. In the first approach, rice tissues were treated with $M$. oryzae mycelial extracts containing the fungal elicitors. Samples from mock- and elicitor-treated rice tissues were collected for miRNA analysis. Candidate miRNAs were those showing altered accumulation between elicitor- and mock-treated samples. Using this method, some conserved miRNAs/miRNA families and novel miRNAs were found to be responsive to the blast fungal elicitors, suggesting their possible involvement in rice- $M$. oryzae interaction (Baldrich et al. 2015). These miRNAs have been shown to target a wide range of 
genes involved in a number of pathways, via degradomics analysis (Baldrich et al. 2015). In the second strategy, rice seedlings were inoculated with a virulent $M$. oryzae strain, following which samples were collected for miRNA analysis. Candidate miRNAs were those showing altered abundance following inoculation with M. oryzae. Using this method, the expression levels of a number of miRNAs were found to be significantly altered in the rice accession Nipponbare (NPB) or its mutant following infection with $M$. oryzae (Zhang et al. 2015; Wang et al. 2018a; Zhang et al. 2018). In the third approach, rice seedlings from one susceptible and one resistant accession were inoculated with a $M$. oryzae strain, with samples subsequently collected for miRNA analysis. Candidate miRNAs were those showing altered abundance between the susceptible and resistant accessions in response to $M$. oryzae infection. Using this method, a subset of miRNAs was found to be differentially expressed in the susceptible and resistant accessions, suggesting their possible roles in rice- $M$. oryzae interaction ( $\mathrm{Li}$ et al. 2014; $\mathrm{Li}$ et al. 2016b; Dong et al. 2018).

To date, more than 70 miRNAs from $61 M I R$ families have been identified to be responsive to $M$. oryzae or its elicitors (Table 1). These miRNAs have been predicted to regulate various signaling pathways by targeting a wide range of genes, including those involved in the biogenesis of small RNAs, auxin signaling, salicylic acid (SA)/jasmonic acid (JA)/ethylene (ET) signaling, and ROS signaling, as well as genes containing upstream open reading frames (Baldrich et al. 2015; Wang et al. 2018a; Zhang et al. 2018; Li et al. 2019a). Intriguingly, candidate miRNAs responsive to $M$. oryzae included members from the miR162 and miR168 families (Campo et al. 2013; Li et al. 2014). It is known that miR162 regulates DCL1 and miR168 controls AGO1 (Wu et al. 2015; Zhang et al. 2015), suggesting that the miRNA signaling pathway plays important roles in rice immunity against $M$. oryzae. The identification of these miRNAs opens up a door to explore the fine-tuning mechanisms underlying rice- $M$. oryzae interaction.

\section{The microRNAs that play positive roles in rice immunity against $M$. oryzae}

To date, members of four miRNA families have been reported to act as positive regulators of rice immunity against $M$. oryzae, namely miR160, miR166, miR398 and miR7695.

The miR160 family is a highly conserved miRNA family, consisting of six loci in the rice genome and being capable of generating three mature isoforms (Table 1). The abundance of each of the three mature isoforms was altered in response to infection by $M$. oryzae or treatment with its elicitors (Campo et al. 2013; Li et al.
2014). The mature miR160a/b/c/d isoform seems to be transcribed mainly from OsMIR160a and OsMIR160b and accumulates to greater concentrations than miR160e and miR160f in leaves ( $\mathrm{Li}$ et al. 2014; Huang et al. 2016a). miR160 targets five Auxin Response Factors $(A R F \mathrm{~s})$ transcription factor genes, namely $A R F 8$ ( $L O C_{-}$ Os02g41800), ARF10 (LOC_Os04g43910), ARF13 (LOC_ Os04g59430), ARF18 (LOC_Os06g47150) and ARF22 (LOC_Os10g33940) (Wu et al. 2009; Li et al. 2010b). Transgenic lines overexpressing miR160a showed reduced susceptibility to $M$. oryzae, a phenotype which was associated with suppression of the expression of target genes such as ARF8, ARF10 and ARF13, indicating that miR160 positively regulates rice blast disease resistance via $A R F$ s that may involve auxin signaling; this finding, is also, consistent with the opinion that auxin signaling antagonizes immunity. However, the mechanism by which suppression of $A R F$ s leads to increased resistance remains to be elucidated.

The miR166 family has 13 members in the rice genome, forming six mature isoforms (Table 1). The function of miR166 has been reported in plant development, targeting transcription factor genes of the class III homeodomain-leucine zipper family, such as $P H A B U$ LOSA and PHABOLUTA, to specify the fate of the shoot apical meristem (Zhu et al. 2011; Li et al. 2019b). Its function in plant immunity has been reported only in rice. The difference in expression between resistant and susceptible accessions or in response to $M$. oryzae or its elicitors varied between individual miR166 members. For example, miR166m was down-regulated following treatment with M. oryzae elicitors (Campo et al. 2013). The expression of miR166k-/l-3p was constitutively higher in the susceptible accession Lijiangxin Tuan Hegu (LTH) than in the resistant accession IRBLkm-Ts, but was up-regulated in both accessions in response to infection by M. oryzae (Li et al. 2014). In the intermediate susceptible/resistant accession NPB, expression of both miR166k-3p and miR166j-3b was up-regulated following M. oryzae infection (Zhang et al. 2018). In a knockdown dcl1 mutant, expression of miR166j-5 was downregulated, whereas, expression of $\mathrm{miR} 166 \mathrm{a} / \mathrm{b} / \mathrm{c} / \mathrm{d}-3 \mathrm{p} / \mathrm{f}$ was up-regulated following $M$. oryzae infection (Zhang et al. 2015). The expression patterns suggest that the functions may differ among different members of the miR166 family. However, in-depth functional investigation has been carried out only with respect to the miR166k-166 h polycistron, that co-expresses miR166k and miR166h (Baldrich et al. 2016). Up-regulation of miR166k and miR166h from an activation mutant miR166k-166h-Ac led to increased resistance to rice blast disease, a phenotype which was associated with activation of the ET-signaling pathway and high marked up-regulation of defense-related genes, although 
Table 1 miRNAs responsive to Magnaporthe oryzae or its elicitors ${ }^{a}$

\begin{tabular}{|c|c|c|c|c|c|}
\hline miRNA & Loci & $\begin{array}{l}\text { Mature } \\
\text { length } \\
\text { (nt) }\end{array}$ & $\begin{array}{l}\text { Mature } \\
\text { isoforms }\end{array}$ & Target gene & Literature \\
\hline miR156 & 13 & 20,21 & 3 & $\begin{array}{l}\text { SPL13 (LOC_Os07g32170); SPL18 (LOC_Os09g32944); SPL19 } \\
\text { (LOC_Os11930370); SPL14 (LOC_Os08g39890); SPL3 (LOC_ } \\
\text { Os02g04680); SPL4(LOC_Os02g07780) }\end{array}$ & $\begin{array}{l}\text { Campo et al. 2013; Li et al. 2014; Baldrich et al. } \\
\text { 2015; Zhang et al. 2015; Li et al. 2016b; Wang } \\
\text { et al. 2018a; Zhang et al. } 2018\end{array}$ \\
\hline miR159 & 6 & 21 & 6 & $\begin{array}{l}\text { GAmyb (LOC_Os01959660, LOC_Os06g40330); Zinc finger } \\
\text { (LOC_Os10g05230) }\end{array}$ & Li et al. 2014; Xu et al. 2014; Li et al. 2016b \\
\hline miR160 & 6 & 21 & 3 & $\begin{array}{l}\text { ARF8 (LOC_Os02g41800); ARF10 (LOC_Os04g43910); ARF13 } \\
\text { (LOC_Os04g59430); ARF18 (LOC_Os06g47150); ARF22 (LOC_ } \\
\text { Os10g33940) }\end{array}$ & $\begin{array}{l}\text { Campo et al. 2013; Li et al. 2014; Xu et al. 2014; } \\
\text { Baldrich et al. 2015; Li et al. 2016b }\end{array}$ \\
\hline miR162 & 2 & 21 & 2 & DCL1a (LOC_Os03g02970) & $\begin{array}{l}\text { Li et al. 2014; Zhang et al. 2015; Li et al. 2016b; } \\
\text { Wang et al. 2018a; Zhang et al. } 2018\end{array}$ \\
\hline miR164 & 6 & 21 & 4 & $\begin{array}{l}\text { MTN4 (LOC_Os06g46270); MTN6 (LOC_Os08g10080); MTN5 } \\
\text { (LOC_Os06g23650); MTN3 (LOC_Os12g41680); RGHIA; Salicylic } \\
\text { acid-induced protein } 19 \text { (LOC_Os12g41680) }\end{array}$ & $\begin{array}{l}\text { Campo et al. 2013; Li et al. 2014; Xu et al. 2014; Li } \\
\text { et al. 2016b; Wang et al. 2018a }\end{array}$ \\
\hline
\end{tabular}

$\operatorname{miR166} 13 \quad 21 \quad 6$

$\operatorname{miR} 167 \quad 10 \quad 21 \quad 2$

$\operatorname{miR} 168 \quad 2 \quad 21,24 \quad 3$

$\operatorname{miR} 169 \quad 17 \quad 21,22 \quad 9$

$\begin{array}{llll}\operatorname{miR} 171 & 9 & 21 & 10\end{array}$

$\operatorname{miR172} 4 \quad 20,21-4$

$\operatorname{miR319} 2 \quad 20,21$

$\operatorname{miR390} 1 \quad 20,21 \quad 2$

$\operatorname{miR393} 221,22 \quad 2$

$\operatorname{miR394} 120 \quad 1$

$\operatorname{miR395} 25 \quad 21,22 \quad 10$

$\operatorname{miR396} \quad 8 \quad 21,22 \quad 5$

$\begin{array}{llll}\operatorname{miR} 398 & 2 & 21 & 2\end{array}$

$\operatorname{miR435} \quad 1 \quad 20 \quad 1$

$\operatorname{miR439} \quad 10 \quad 21 \quad 1$
START domain-containing protein (LOC_Os03g01890); Homeobox-leucine zipper protein (LOC_Os03g43930, LOC_ Os10g33960, LOC_Os12g41860); Vacuolar protein 8 (LOC_ Os06901304)

ARF6 (LOC_Os02g06910); ARF12 (LOC_Os04g57610); ARF17 (LOC_Os06g46410); ARF25 (LOC_Os12g41950); Retinol dehydrogenase 14 (LOC_Os06g03830); Zinc finger protein 207 (LOC_Os09g38790)

AGO1a (LOC_Os02g45070); AGO1b (LOC_Os04g47870); AGO1C (LOC_Os02g58490); AGO1d (LOC_Os06g51310)

NF-YC1 (LOC_Os02g07450); NF-YC2 (LOC_Os03g 14669); NF-YC3 (LOC_Os04g58680); NF-YC4 (LOC_Os06945640); NF-YA11 (LOC_ Os02g53620); NF-YA10 (LOC_Os 12g42400) Os04g44360, LOC_Os04g46860); AP2 domain containing TF (ERF\#043-ERF\#073-ERF\#090)

Retrotransposon protein (LOC Os05943220); SSH1 (LOC Os07g13170); ARF9 (LOC_Os04g36054); AP2-like factor (LOC_ Os03960430, LOC Os04955560, LOC Os05g03040, LOC Os07913170); Putative zinc-finger motif (LOC_Os09g21770)

TCP21 (LOC_Os12g07480); myb proto-oncogene protein (LOC_ Os01g59660)

STRUBBELIG-RECEPTOR FAMILY 6 (LOC_Os03951040); Wallassociated receptor kinase-like 10 (LOC_Os04g30060); Leucinerich repeat family protein (LOC_Os04g45170)

TIR1 (LOC_Os05g05800); AFB2 (LOC_Os04g32460)

FBX32 (LOC_Os01g69940); RNA polymerase sigma factor rpoD (LOC_Os05g51150)

Cytochrome b5-like Heme/steroid binding domain containing protein (LOC_Os10g35870); Bifunctional 3-phosphoadenosine 5phosphosulfate synthetase (LOC_Os03953230); Protein kinase domain containing protein (LOC_Os05g44290)

GRF6 (LOC_Os03951970); GRF7 (LOC_Os 12g29980); GRF8 (LOC Os11935030); GRF9 (LOC_Os03g47140); GRF2 (LOC Os06g 10310); GRF6 (LOC_Os03951970); Deaminase (LOC_ Os06g29430)

CSD1 (LOC_Os03g22810), CSD2 (LOC_Os07g46990), CSD3 (LOC_Os03g11960), CSD4 (LOC_Os08g44770)

COBRA-like protein precursor (LOC_Os07g41320) (predicted)

Ch19 (LOC_Os03936540); Cyclin-dependent kinase G-1 (LOC_ Os02g39010)
Campo et al. 2013; Li et al. 2014; Xu et al. 2014; Zhang et al. 2015; Li et al. 2016b; Zhang et al. 2018

Liu et al. 2012; Campo et al. 2013; Li et al. 2014; Xu et al. 2014; Zhang et al. 2015; Li et al. 2016b; Wang et al. 2018a

Campo et al. 2013; Li et al. 2014; Zhang et al. 2015; Li et al. 2016b

Campo et al. 2013; Li et al. 2014; Zhang et al. 2015; Li et al. 2016b; Wang et al. 2018a; Zhang et al. 2018

Campo et al. 2013; Li et al. 2014; Xu et al. 2014; Baldrich et al. 2015; Li et al. 2016b

Li et al. 2014; Xu et al. 2014; Baldrich et al. 2015; Li et al. 2016b; Wang et al. 2018a

Xu et al. 2014; Li et al. 2016b; Zhang et al. 2018

Campo et al. 2013; Li et al. 2016b

Campo et al. 2013; Li et al. 2014; Baldrich et al. 2015; Li et al. 2016b

Campo et al. 2013; Li et al. 2016b; Wang et al. $2018 a$

Xu et al. 2014; Baldrich et al. 2015; Li et al. 2016b

Campo et al. 2013; Li et al. 2014; Zhang et al. 2015; Li et al. 2016b

Campo et al. 2013; Li et al. 2014; Li et al. 2016b; Wang et al. 2018a

Li et al. 2014; Baldrich et al. 2015; Li et al. 2016b; Zhang et al. 2018

Li et al. 2014; Xu et al. 2014; Li et al. 2016b 
Table 1 miRNAs responsive to Magnaporthe oryzae or its elicitors ${ }^{\mathrm{a}}$ (Continued)

\begin{tabular}{|c|c|c|c|c|c|}
\hline miRNA & Loci & $\begin{array}{l}\text { Mature } \\
\text { length } \\
\text { (nt) }\end{array}$ & $\begin{array}{l}\text { Mature } \\
\text { isoforms }\end{array}$ & Target gene & Literature \\
\hline miR444 & 6 & 21 & 6 & $\begin{array}{l}\text { MADS23 (LOC_Os08g33488); MADS27 (LOC_Os02g36924); } \\
\text { MADS57 (LOC_Os02g49840) }\end{array}$ & $\begin{array}{l}\text { Campo et al. 2013; Li et al. 2014; Li et al. 2016b; } \\
\text { Zhang et al. } 2018\end{array}$ \\
\hline miR528 & 2 & 21 & 2 & $\begin{array}{l}\text { Laccase (LOC_Os01g62600); Copper ion binding protein (LOC_ } \\
\text { Os01g03620, LOC_Os01g03640) }\end{array}$ & Campo et al. 2013; Li et al. 2014; Li et al. 2016b \\
\hline miR529 & 2 & 20,21 & 2 & SBP-box gene family member (LOC_Os02g07780) & $\begin{array}{l}\text { Campo et al. 2013; Baldrich et al. 2015; Li et al. } \\
\text { 2016b }\end{array}$ \\
\hline miR530 & 1 & 20 & 1 & $\begin{array}{l}\text { Transcriptional regulator (LOC_Os05g34720); Expression protein } \\
\text { (LOC_Os01g52920); Cyclin-T1-2; LORICRIN }\end{array}$ & Li et al. 2014; Baldrich et al. 2015; Li et al. 2016b \\
\hline miR535 & 1 & 21 & 2 & $\begin{array}{l}\text { Expressed protein (LOC_OsO2g09080, LOC_OsO3g 14880); } \\
\text { Squamosa promoter-binding-like protein } 11 \text { (LOC_OsO6g45310) }\end{array}$ & Li et al. 2014; Xu et al. 2014; Li et al. 2016b \\
\hline miR810 & 2 & 21 & 3 & $\begin{array}{l}\text { FBX160 (LOC_Os05g07950); Retrotransposon protein (LOC_ } \\
\text { Os02g38610) (predicted) }\end{array}$ & Baldrich et al. 2015; Li et al. 2016b \\
\hline miR812 & 22 & $\begin{array}{l}21,22, \\
24\end{array}$ & 15 & $\begin{array}{l}\text { Calcium/calmodulin dependent protein kinase (LOC_ } \\
\text { Os03g22050) }\end{array}$ & Li et al. 2014; Li et al. 2016b \\
\hline miR818 & 6 & 21,22 & 3 & RDR2 (LOC_Os04g39160) & Baldrich et al. 2015; Li et al. 2016b \\
\hline miR820 & 3 & 21 & 1 & $\begin{array}{l}\text { DRM2 (LOC_Os03g02010); Cellulose synthase like C12 (LOC_ } \\
\text { OS11g13650) }\end{array}$ & Campo et al. 2013; Li et al. 2016b \\
\hline miR827 & 2 & 21 & 2 & $\begin{array}{l}\text { SPX-MFS1 (LOC_Os04g48390); SPX-MFS2 (LOC_Os02g45520); } \\
\text { WAK receptor-like protein kinase (LOC_Os02g56370) }\end{array}$ & Campo et al. 2013; Li et al. 2014; Li et al. 2016b \\
\hline miR1319 & 2 & 24 & 2 & LTPL6 (LOC_Os10g05720) & Baldrich et al. 2015; Li et al. 2016b \\
\hline $\operatorname{miR} 1320$ & 1 & 21 & 1 & $\begin{array}{l}\text { Ethylene-responsive transcription factor (LOC_Os10g41330); } \\
\text { ANTHOCYANIDIN 3-O-glucosyltransferase }\end{array}$ & Li et al. 2014; Wang et al. 2018a \\
\hline miR1423 & 2 & 21,24 & 2 & $\begin{array}{l}\text { Expressed protein (LOC_Os11g28540); Heat shock protein DnaJ } \\
\text { (LOC_Os06g09560) }\end{array}$ & Baldrich et al. 2015; Li et al. 2016b \\
\hline $\operatorname{miR} 1425$ & 1 & 21 & 1 & $\begin{array}{l}\text { Rf4 (LOC_Os10g35240); Rf6 (LOC_Os10g35436); Protein kinase } \\
\text { (LOC_Os01g49614) }\end{array}$ & $\begin{array}{l}\text { Campo et al. 2013; Li et al. 2014; Baldrich et al. } \\
\text { 2015; Li et al. 2016b }\end{array}$ \\
\hline miR1427 & 1 & 21 & 1 & YGL8 (LOC_Os01g17170) & Xu et al. 2014; Baldrich et al. 2015; Li et al. 2016b \\
\hline $\operatorname{miR} 1430$ & 1 & 21 & 1 & $\begin{array}{l}\text { ASYMMETRIC LEAVES 2 (LOC_Os05g34450); myb/SANT domain } \\
\text { protein (LOC_Os03g13790) }\end{array}$ & Campo et al. 2013; Li et al. 2016b \\
\hline miR1433 & 1 & 21 & 1 & $\begin{array}{l}\text { NAD dependent epimerase/dehydratase family protein (LOC_ } \\
\text { Os09g15420), Retrotransposon protein (LOC_Os04g17640) } \\
\text { (predicted) }\end{array}$ & Li et al. 2014; Li et al. 2016b \\
\hline $\operatorname{miR} 1437$ & 2 & $\begin{array}{l}21,22, \\
24\end{array}$ & 3 & $\begin{array}{l}\text { Ubiquitin carboxyl-terminal hydrolase domain containing pro- } \\
\text { tein (LOC_Os06g0184300); FAD2-1(LOC_Os02g48560) }\end{array}$ & Xu et al. 2014; Baldrich et al. 2015; Li et al. 2016b \\
\hline miR1846 & 5 & $\begin{array}{l}20,21 \\
22\end{array}$ & 3 & $\begin{array}{l}\text { Expression protein (LOC_Os08g10350); Anther-specific proline- } \\
\text { rich protein (LOC_OsO2g18870); Zinc-finger, C3H4 type do- } \\
\text { main containing TF }\end{array}$ & Baldrich et al. 2015; Li et al. 2016b \\
\hline miR1847 & 2 & 21,24 & 2 & AGO4b (LOC_Os04g06770) & Baldrich et al. 2015; Li et al. 2016b \\
\hline miR1850 & 3 & 21,22 & 3 & $\begin{array}{l}\text { Pectinesterase inhibitor domain (LOC_Os08g04650); RDR2; NB- } \\
A R C\end{array}$ & Campo et al. 2013; Li et al. 2016b \\
\hline miR1854 & 1 & 21,22 & 2 & $\begin{array}{l}\text { Glucose-6-phosphate 1-dehydrogenase (LOC_Os03g20300) } \\
\text { (predicted) }\end{array}$ & Baldrich et al. 2015; Li et al. 2016b \\
\hline $\operatorname{miR} 1858$ & 2 & 21 & 1 & $\begin{array}{l}\text { CSLC9-cellulose synthase-like family C (LOC_Os03g56060) } \\
\text { (predicted) }\end{array}$ & Baldrich et al. 2015; Li et al. 2016b \\
\hline miR1861 & 15 & 22 & 8 & $\begin{array}{l}\text { Starch binding domain containing protein (LOC_Os01g63810); } \\
\text { ATP binding protein (LOC_Os05g51790) }\end{array}$ & Li et al. 2014; Baldrich et al. 2015; Li et al. 2016b \\
\hline miR1862 & 7 & 20,24 & 4 & SART-1 family protein (LOC_Os02g30730) & Zhang et al. 2015; Li et al. 2016b \\
\hline miR1863 & 3 & 23,24 & 4 & Predicted expression protein (LOC_Os05g 13804) & Li et al. 2014; Li et al. 2016b; Wang et al. 2018a \\
\hline miR1865 & 1 & 24 & 2 & Aspartate aminotransferase (LOC_OsO2g 14110) & Campo et al. 2013; Li et al. 2016b \\
\hline miR1867 & 1 & 24 & 1 & tRNA methyltransferase (LOC_Os10g30550) & Li et al. 2014; Li et al. 2016b \\
\hline miR1871 & 1 & 24 & 1 & Ribosomal protein L7Ae (LOC_Os09g32976) & Li et al. 2014; Li et al. 2016b \\
\hline
\end{tabular}


Table 1 miRNAs responsive to Magnaporthe oryzae or its elicitors ${ }^{\mathrm{a}}$ (Continued)

\begin{tabular}{|c|c|c|c|c|c|}
\hline miRNA & Loci & $\begin{array}{l}\text { Mature } \\
\text { length } \\
(\mathrm{nt})\end{array}$ & $\begin{array}{l}\text { Mature } \\
\text { isoforms }\end{array}$ & Target gene & Literature \\
\hline miR1874 & 1 & 24 & 2 & Expression protein (LOC_OsO2g20950) & Li et al. 2014; Baldrich et al. 2015; Li et al. 2016b \\
\hline miR1876 & 1 & 24 & 1 & Esterase/lipase/thioesterase (LOC_Os02g31200) & Campo et al. 2013; Li et al. 2016b \\
\hline miR1879 & 1 & 24 & 1 & Catalase isozyme B (LOC_Os06g51150) & Campo et al. 2013; Li et al. 2016b \\
\hline miR1882 & 8 & 24 & 2 & $\begin{array}{l}\text { ABC transporter, ATP-binding protein (LOC_Os11g39020) } \\
\text { (predicted) }\end{array}$ & Zhang et al. 2015; Li et al. 2016b \\
\hline $\operatorname{miR} 2871$ & 2 & 21 & 1 & $\begin{array}{l}\text { Glycosyltransferase family } 43 \text { protein (LOC_Os10g13810); Zinc } \\
\text { finger, C3HC4 type, domain containing TF }\end{array}$ & Xu et al. 2014; Baldrich et al. 2015 \\
\hline $\operatorname{miR} 2873$ & 3 & 21,24 & 3 & TKL_IRAK_CrRLK1L_1.4 (LOC_Os01g06280) (predicted) & Li et al. 2014; Li et al. 2016b \\
\hline $\operatorname{miR} 2878$ & 1 & 24 & 2 & Plastocyanin-like domain containing protein (LOC_Os08g37670) & Li et al. 2014; Li et al. 2016b \\
\hline miR5150 & 1 & 24 & 2 & Receptor-like protein kinase 2 precursor (LOC_Os01g70260) & Li et al. 2016b; Zhang et al. 2018 \\
\hline miR5153 & 1 & 24 & 1 & $\begin{array}{l}\text { NHX1 (LOC_Os07g47100); Chloroplast ribonuclease III domain } \\
\text { protein (LOC_Os01g59510) }\end{array}$ & Li et al. 2016b; Wang et al. 2018a \\
\hline miR5794 & 1 & 21 & 1 & $\begin{array}{l}\text { Rapid ALkalinization Factor14 (RALF14, LOC_Os11g26880); } \\
\text { RALF4 (LOC_Os12g35670) }\end{array}$ & Zhang et al. 2015; Li et al. 2016b \\
\hline miR7695 & 1 & 21,24 & 5 & OsNramp6 (LOC_Os01g31870) & Campo et al. 2013 \\
\hline
\end{tabular}

${ }^{a}$ This list only includes those miRNAs that are functionally characterized or reported by two or more papers

miR166k-5p suppresses the expression of two OsEIN2 genes (Salvador-Guirao et al. 2018). This finding was consistent with an involvement of ET signaling in rice immunity against M. oryzae (Yang et al. 2017), although it was unclear how suppression of OsEIN2s led to activation of the ET-signaling pathway.

The miR398 family has two members in rice genome, generating two mature isoforms (Table 1). This family is highly conserved in both monocotyledons and dicotyledons, including rice, wheat, barley, switchgrass, Arabidopsis, Brassica rapa and Populus trichocarpa. miR398 targets four members of the 15 superoxidase dismutase (SOD) gene family in rice, i.e., CSD1, CSD2, SODX and CCSD (Li et al. 2019a). Overexpression of miR398b led to increased resistance to the rice blast disease, in association with the down-regulation of all four target genes, up-regulation of defense-related genes and increased accumulation of $\mathrm{H}_{2} \mathrm{O}_{2}$ ( $\mathrm{Li}$ et al. 2014). By contrast, blocking miR398b by overexpressing a target gene mimic resulted in increased susceptibility, which was associated with up-regulation of all four target genes, delayed induction of defense-related genes and reduced $\mathrm{H}_{2} \mathrm{O}_{2}$ accumulation ( $\mathrm{Li}$ et al. 2019a). Detailed analysis indicated that CSD1, CSD2 and SODX negatively regulated $\mathrm{H}_{2} \mathrm{O}_{2}$ accumulation, whereas CCSD positively regulated it; the loss-of-function mutants $c s d 1$, $\operatorname{cs} d 2$ and $\operatorname{sodx}$ exhibited increased susceptibility. CSD1, $C S D 2$ and SODX appear to determine the production of $\mathrm{H}_{2} \mathrm{O}_{2}$ by coordinately regulating the expression of the other $S O D$ family members and hence the total SOD enzyme activity. In contrast, CCSD is probably required for CSD enzyme activity because the ccsd mutant exhibited little CSD activity (Li et al. 2019a). Therefore, miR398 fine-tunes ROS signaling through these four $S O D$ members to regulate rice immunity against $M$. oryzae. Nevertheless, the function of miR398 in rice immunity obviously differs from those in Arabidopsis and barley, where miR398 negatively regulates immunity to the bacterial pathogen Pst DC3000 and the powdery mildew pathogen Blumeria graminis f. sp. hordei, respectively (Li et al. 2010a; Xu et al. 2014). Therefore, the detailed functions of miR398 need to be further investigated in different phytopathological systems.

miR7695 was the first miRNA to be identified as being responsive to the elicitors of $M$. oryzae, and is expressed in leaves throughout the rice vegetative growth stage (Campo et al. 2013). Its abundance is reduced in the $d c l 4$, but not in $d c l 1$ mutant, indicating that MIR7695 is a recently evolved $M I R$ locus, the primary transcript of which is processed by $D C L 4$, producing multiple miRNAs (Campo et al. 2013). Indeed, five mature miR7695s were detected by northern blotting analysis (Campo et al. 2013). Mature miR7695.3-3p targets one transcript of NRAMP6 (Natural resistance-associated macrophage protein 6), a locus that generates eight transcripts as a result of alternative splicing (Campo et al. 2013). miR7695 seems to occur specifically in rice, particularly in the japonica subspecies and in some indica accessions. Its expression is higher in blast-resistant accessions than in blast-susceptible ones, and is further upregulated in response to infection by $M$. oryzae (Quoc et al. 2019). Overexpression of its precursor leads to increased resistance to $M$. oryzae. Therefore, miR7695 plays positive roles in rice immunity against $M$. oryzae. Consistent with this role of miR7695, loss-of-function mutants of its target gene NRAMP6 exhibited reduced 
plant growth but increased resistance to $M$. oryzae (Peris-Peris et al. 2017). NRAMP6 encodes a metal transporter that has iron and manganese transport activity. Therefore, it is presumed that NRAMP6 acts via metal homeostasis, linking immunity to $M$. oryzae to rice plant growth, a process which is fine-tuned by miR7695.

Taken together, some miRNAs played positive roles in regulating rice immunity against $M$. oryzae via regulating a number of signaling pathways, such as auxin signaling, ethylene signaling, ROS signaling and metal homeostasis.

\section{The microRNAs that facilitate infection by M. oryzae in} rice

Currently, members of six miRNA families have been reported to facilitate the infection by $M$. oryzae, namely miR164, miR167, miR169, miR319, miR396 and miR444.

The miR164 family is a highly conserved miRNA family containing six loci, which generate four mature isoforms, targeting six genes encoding NAC (NAM, ATAF1/2 and CUC2) transcription factors in rice (Fang et al. 2014). The miR164-NAC regulatory module functions in ET-signaling to control leaf senescence in Arabidopsis and drought tolerance in rice ( $\mathrm{Li}$ et al. 2013; Fang et al. 2014). Recently, evidence has emerged of its roles in rice- $M$. oryzae interactions. The abundance of $\mathrm{miR} 164 \mathrm{a} / \mathrm{b} / \mathrm{f}$ was altered in response to infection by $M$. oryzae or treatment with the blast fungus elicitors (Campo et al. 2013; Li et al. 2014; Wang et al. 2018a). Overexpressing miR164a led to increased susceptibility to $M$. oryzae, failing to up-regulate the expression of defense-related genes and $\mathrm{H}_{2} \mathrm{O}_{2}$ production following infection by $M$. oryzae (Wang et al. 2018a). Consistently, mutation in one target gene, OsNAC60, resulted in increased susceptibility to $M$. oryzae. In contrast, transient overexpression of OsNAC60 activated cell death that was associated with $\mathrm{H}_{2} \mathrm{O}_{2}$ production in Nicotiana benthamiana (Wang et al. 2018a). Therefore, miR164 negatively regulated rice immunity to $M$. oryzae via at least one of its targeted $N A C$ transcription factor genes coupled to ET signaling. However, it is unclear whether the other target genes were involved in miR164mediated regulation of rice- $M$. oryzae interaction.

In rice, the miR167 family has 10 members, forming two mature isoforms that target four Auxin Responsive Factors and three other genes, including one encoding a NLR family protein (Zhao et al. 2019). Over-expressing miR167d resulted in increased susceptibility to $M$. oryzae in a resistant accession genetic background, a phenomenon that was associated with failure to induce defense-related genes and consequent reduced $\mathrm{H}_{2} \mathrm{O}_{2}$ production (Zhao et al. 2019). In contrast, blocking miR167d by expressing a target mimic led to increased resistance to $M$. oryzae, associated with high expression of defense-related genes and increased $\mathrm{H}_{2} \mathrm{O}_{2}$ production.
The miR167d-mediated regulation of immunity seemed to be associated with neither ARF25 (LOC_Os12g41950) nor the gene encoding the NLR protein ( $L O C_{-}$ Os07g29820), because knocking-out ARF25 or overexpressing or knocking-out of LOC_OsO7g29820 did not generate any obvious change in the rice blast disease phenotype (Zhao et al. 2019). In contrast, knocking-out of ARF12 led to increased susceptibility to $M$. oryzae (Zhao et al. 2019), implying that miR167d mediates its functions via $A R F 12$. In addition, the miR167d-mediated regulation of rice- $M$. oryzae interaction may be associated with both IAA- and JA-signaling pathways, because JA concentration decreased in transgenic lines overexpressing miR167d, but increased in those overexpressing the target mimicry (Zhao et al. 2019). Therefore, it would be interesting to investigate the context of the connection between ARF12 and IAA/JA signaling in terms of rice immunity against $M$. oryzae.

miR169 is a relatively large miRNA family containing 17 members, which generate nine mature isoforms in rice, targeting eight of the 11 nuclear transcription factor $Y$ subunit A (NF-YA) genes (Wu et al. 2009; Li et al. 2010b). NF-YA, together with NF-YB and NF-YC, forms a heterotrimer to control the expression of downstream genes. The different isoforms of miR169 show considerable variation in expression patterns and are differentially responsive to $M$. oryzae or its elicitors (Campo et al. 2013; Li et al. 2014; Li et al. 2017). However, they may play negative roles in rice immunity against $M$. ory$z a e$, because the total miR169 abundance was significantly up-regulated in the susceptible rice accession in response to $M$. oryzae, but varied only slightly in the resistant accession in response to infection by $M$. oryzae (Li et al. 2017). Consistent with this observation, overexpression of miR169a resulted in increased susceptibility to $M$. oryzae, which was associated with compromised defense responses, such as reduced induction of defenserelated genes and decreased $\mathrm{H}_{2} \mathrm{O}_{2}$ production ( $\mathrm{Li}$ et al. 2017). In contrast, blocking miR169 via expressing a target mimicry of miR169a led to increased resistance to $M$. oryzae, which may be attributed to up-regulation of some or all of the eight target NF-YAs (Li et al. 2017). However, it remains to be determined whether (or which of) the target $N F-Y A$ s positively regulates rice immunity against $M$. oryzae and which signal transduction pathway is involved.

The miR319 family includes two members, which form one mature miRNA targeting teosinte branched/cycloidea/ proliferation cell factors 21 (TCP21) and the positive regulator of gibberellin (GA) signaling, GAMYB, in rice (Zhang et al. 2018). The expression of miR319 was up-regulated, whereas that of its target genes was down-regulated in response to $M$. oryzae infection in a susceptible accession (Zhang et al. 2018). Similarly, a miR319-resistant TCP21 
mutant exhibited resistance to $M$. oryzae, whereas knockdown of TCP21 led to increased susceptibility to $M$. oryzae. The resistant phenotypes were associated with upregulation of expression of genes involved in JA biosynthesis/signaling, marked up-regulation of defense-related genes and increased accumulation of $\mathrm{H}_{2} \mathrm{O}_{2}$. In contrast, the susceptible phenotypes were associated with downregulation of genes involved in JA biosynthesis/signaling and a failure of $\mathrm{H}_{2} \mathrm{O}_{2}$ production (Zhang et al. 2018). Therefore, miR319 probably regulates rice immunity against $M$. oryzae by TCP21-mediated regulation of JA biosynthesis and signaling.

The miR396 family is a highly conserved and abundant miRNA family. It consists of eight members in the rice genome, forming five mature isoforms, which target 11 Growth Regulating Factor (GRF) transcription factor genes (Duan et al. 2015; Gao et al. 2015; Li et al. 2016a). The miR396-GRF module has been reported to finetune inflorescence development controlling panicle and individual grain size (Tang and Chu 2017). Overexpressing miR396a, miR396c, miR396d or miR396h led to increased susceptibility to $M$. oryzae, which was associated with reduced $\mathrm{H}_{2} \mathrm{O}_{2}$ production and a failure to induce defense-related genes (Chandran et al. 2019). In contrast, blocking miR396 by expressing a target mimicry of miR396d or a short tandem target mimicry (STTM) of miR396d-396e led to increased resistance to $M$. oryzae, which was associated with marked up-regulation of defense-related genes and increased $\mathrm{H}_{2} \mathrm{O}_{2}$ production. Consistent with these being the target genes of miR396, overexpression of GRF6, GRF7, GRF8 or GRF9 resulted in increased resistance to $M$. oryzae, whereas, knock-down of GRF7 via RNA interference (RNAi) led to increased susceptibility (Chandran et al. 2019). Thus, miR396 facilitates the infection by $M$. oryzae via at least four GRF genes. Because blocking miR396 led to improvements in both rice yield and immunity against $M$. oryzae, the miR396-GRF module is invaluable in breeding programs aiming to improve both of yield and disease resistance simultaneously.

The miR444 family is a monocot-specific miRNA family with six gene loci in the rice genome, each generating 1-3 mature miRNAs, leading to six mature isoforms (Table 1). miR444 targets four MADS-box transcription factor genes and four other genes (Wu et al. 2009; Li et al. 2010b), and plays roles in root and tiller development, as well as in response to Rice stripe virus (RSV) (Yan et al. 2014; Wang et al. 2016). Overexpression of miR444s resulted in increased resistance to RSV that was associated with up-regulation of OsRDR1 expression via suppression of the miR444-targeted MADS genes (Wang et al. 2016), indicating that miR444a plays a positive role in resistance to the virus. On the other hand,
miR444b seemed to play negative roles with respect to rice immunity against $M$. oryzae. In response to $M$. ory$z a e$ infection, the abundance of miR444b.2 was increased in a blast-susceptible rice accession, but was decreased in a blast-resistant accession (Xiao et al. 2017). In contrast, expression of the target genes of miR444b.2, including MADS27b and MADS57, changed only slightly in the susceptible accession but increased significantly in the resistant accession, indicating that miR444b facilitates the infection by $M$. oryzae via impact on these target genes. In addition, overexpression of miR444b resulted in increased susceptibility to $M$. oryzae; whereas blocking miR444b.2 via overexpression of a target mimicry of miR444b.2 resulted in increased rice blast disease resistance (Xiao et al. 2017). However, the identity of the target genes, which contribute to miR444b-mediated facilitation of infection by $M$. oryzae, has still to be confirmed.

Taken together, the signaling pathways associated with rice defense against the blast disease were also regulated by the miRNAs that facilitate infection of $M$. oryzae, including auxin signaling, ET signaling, JA signaling and some yet-to-be identified signaling pathways, which may also regulate the trade-off between plant growth and immunity.

\section{Challenges facing functional investigations into the roles of miRNAs in rice-M. oryzae interaction}

It is quite challenging to investigate the roles of miRNAs in rice- $M$. oryzae interaction due to the complicated miRNA regulatory nature. Usually, one mature miRNA can be transcribed from one or more MIR gene loci, whereas one gene locus may differ from another in terms of response to $M$. oryzae. One mature miRNA may suppress the expression of one or several target genes and such suppression may vary among different target genes. Moreover, one target gene can be regulated by multiple miRNAs. However, the most challenging problem is to identify the authentic target genes in planta that contribute to the miRNA-mediated phenotypes, because functional analysis of the target genes is essential for an investigation of a miRNA-mediated regulatory mechanism. In this section, we briefly summarize methods reported to achieve the identification of target genes.

The strategy for target gene identification is based on how the miRNA regulates the expression of its target genes. Most plant miRNAs regulate target gene expression via mRNA cleavage. In such a situation, the target genes can be relatively and easily predicted and experimentally validated via degradome sequencing and/or RNA ligase-mediated 5'-rapid amplification of cDNA ends (RLM-5'-RACE) (German et al. 2008). Because miRNA-mediated mRNA cleavage generates 3' decay 
products that have a $3^{\prime}$ poly(A) tail and a $5^{\prime}$ monophosphate end, the $5^{\prime}$ monophosphate ends can be ligated to an adaptor sequence by an RNA ligase. The ligated products can then be reverse-transcribed into cDNA and subjected to high-throughput sequencing or 5'RACE analysis (Wu et al. 2009). However, this strategy is invalid for those target genes that are translationally inhibited by miRNAs or where target site-methylation is mediated by miRNAs. In such cases, the target genes can first be predicted based on sequence complementarity, using online programs such as psRNATarget (http:// plantgrn.noble.org/psRNATarget/) or psRobot (http:// omicslab.genetics.ac.cn/psRobot/) (Wu et al. 2012; Dai et al. 2018). The predicted target genes can be confirmed via a reporter assay, in which the reporter gene is constructed by fusing the predicted target site to a fluorescence protein such as the Yellow Fluorescence Protein (YFP). Then, the reporter gene is transiently expressed or co-expressed with the miRNA. If the predicted target site is authentic, the YFP signal should be weaker or the YFP protein concentration lower as a result of coexpression with miRNA than that from expression of the reporter alone (Li et al. 2017). The reporter assay approach should be valid for targets regulated by miRNAmediated cleavage and translational inhibition, but may not be valid for identification of target genes regulated by miRNA-mediated methylation. In the latter case, the predicted target sites could be confirmed by examining the DNA methylation status via bisulfite sequencing analysis (Wu et al. 2010). After testing each of the above strategies, it may still be impossible to confirm the predicted target genes. Under such circumstances, a combination of the above strategies may be appropriate, with multiple omics analyses, by comparing the transgenic line overexpressing the miRNA with the transgenic line overexpressing its target mimicry with the authentic target genes being among the predicted target genes showing opposite expression patterns in the two transgenic lines. Finally, even if we identified the authentic target genes, we may still face the problem that the target genes identified did not contribute to the miRNAmediated phenotypes. Therefore, the process can be very challenging. Fortunately, most of the blast fungusresponsive miRNAs have known target genes, the regulatory roles of which have been confirmed in a variety of biological processes, such as growth and development (Table 1) (Tang and Chu 2017).

\section{Conclusions}

At present, our understanding of the miRNA-mediated regulation of rice-M. oryzae interaction is quite limited and fragmentary. In the context of the rice- $M$. oryzae interaction, the activation of either PTI or ETI, or both, could lead to either activation or silencing of certain subsets of MIR genes, which, in turn, could recruit networks of downstream genes that may achieve feedback regulation of PTI and/or ETI (Fig. 1). On the one hand, the key component genes of the miRNA signaling pathway, such as DCLs and $A G O$ s, are regulated during PTI and/or ETI, which, in turn, mediate the engagement of the miRNA network in the regulation of rice-M. oryzae interaction. Evidence supporting this conclusion comes from DCL1 and AGO4, in that DCL1 negatively and $A G O 4$ positively regulates rice immunity against $M$. oryzae (Zhang et al. 2015; Deng et al. 2017; SalvadorGuirao et al. 2019). On the other hand, a subset of $M I R$ genes may be down- or up-regulated during PTI and/or ETI, depending on their roles as negative or positive regulators of PTI and/or ETI, whereas the positive regulators would be up-regulated during PTI and/or ETI, the negative regulators would be down-regulated. Evidence supporting this conclusion comes from functionally characterized miRNAs, including miR160, miR164, miR166, miR167, miR169, miR319, miR396, miR398, miR444 and miR7695 (Campo et al. 2013; Li et al. 2014; Li et al. 2017; Xiao et al. 2017; Wang et al. 2018a; Zhang et al. 2018; Chandran et al. 2019; Quoc et al. 2019; Zhao et al. 2019). However, we still do not know how PTI and/or ETI is coupled with the miRNA signaling pathway or with each of the blast fungus-responsive miRNAs.

Some miRNAs may be involved in either PTI or ETI, or both, according to their response to M. oryzae or its elicitors. For example, miR398b may act in PTI, because its abundance was up-regulated in both the susceptible and resistant accessions following $M$. oryzae infection, with overexpression of miR398b leading to increased induction of the PTI marker genes OsKS4 and OsNAC4 in response to PAMP treatment ( $\mathrm{Li}$ et al. 2014). However, current data cannot exclude the involvement of miR398 in ETI. Nevertheless, the miRNA-mediated regulatory nature bestows miRNA with the potential to simultaneously regulate multiple genes. For example, miR169 targets eight of the NF-YA family transcription factor genes and miR396 targets eleven of the GRF genes. In such cases, blocking or overexpressing a single miRNA can achieve up- or down-regulation of multiple genes, facilitating gene manipulation when these target genes have functional redundancy. For example, in tomato, blocking miR482 and miR2118 via STTM resulted in increased resistance to at least two different pathogens (CantoPastor et al. 2019). Therefore, it would be worthwhile investigating the roles of miRNAs in rice- $M$. oryzae interaction and applying them to breeding programs to improve agronomic traits such as disease resistance.

Taken together, progress is clearly being made with respect to unveiling the roles of miRNAs in conferring rice immunity against $M$. oryzae. The future focus should be 
on dissecting the functions of key components in the appropriate miRNA signaling pathway and of each blast fungus-responsive miRNA in the rice- $M$. oryzae interactions. It is highly anticipated that, through MIR genes manipulation we can improve important agronomic traits with lower fitness costs.

\begin{abstract}
Abbreviations
AGO: Argonaute; ARF: Auxin response factor; CCSD: Copper chaperone for superoxide dismutase; CEBiP: Chitin elicitor binding protein; CERK1: Chitin elicitor receptor kinase; CSD: Cu/Zn-superoxidase dismutase; DCL: Dicer-like; ETI: Effector-triggered immunity; GRF: Growth regulating factor; HEN1: Hua enhancer 1; LTH: Lijiangxin Tuan Hegu; LYP: Lysin motif-containing protein miRISC: miRNA-induced silencing complex; NF-YA: Nuclear transcription factor $Y$ subunit A; NLR: Nucleotide-binding site leucine-rich repeat; NRAMP 6: Natural resistance-associated macrophage protein 6; PAMP: Pathogenassociated molecular pattern; PTI: PAMP-triggered immunity; ROS: Reactive oxygen species; SOD: Superoxide dismutase
\end{abstract}

\section{Acknowledgements}

We are grateful to Ji-Qun Zhao, Mei Pu and Dr. Yan-Yan Huang for management of the miRNA-related projects in the lab. We apologize to all colleagues whose work could not be cited due to space limitations.

\section{Authors' contributions}

W-MW, YL and JMJJ wrote the manuscript. YL and W-MW drew the Fig. QF Z-XZ and JMJJ collected data for the table. MIK and JF edited the manuscript. All authors read and approved the final manuscript.

\section{Funding}

This work was supported by the National Natural Science Foundation of China grants (31430072 and 31672090 to W-MW, and 31471761 to YL).

\section{Availability of data and materials}

Not applicable.

\section{Ethics approval and consent to participate}

Not applicable.

\section{Consent for publication}

Not applicable.

\section{Competing interests}

The authors declare that they have no competing interests.

\section{Author details}

'Rice Research Institute and Research Center for Crop Disease and Insect Pests, Sichuan Agricultural University at Wenjiang, 211 Huimin Road, Wenjiang District, Chengdu 611130, China. ${ }^{2}$ Department of Plant Protection, College of Agronomy, Sichuan Agricultural University, 211 Huimin Road, Wenjiang District, Chengdu 611130, China. ${ }^{3}$ Department of Plant Protection, Sindh Agriculture University, Tandojam 70060, Pakistan.

\section{Received: 9 August 2019 Accepted: 28 October 2019}

Published online: 18 November 2019

\section{References}

Abe M, Yoshikawa T, Nosaka M, Sakakibara H, Sato Y, Nagato Y, et al. WAVY LEAF1, an ortholog of Arabidopsis HEN1, regulates shoot development by maintaining microRNA and trans-acting small interfering RNA accumulation in rice. Plant Physiol. 2010;154:1335-46.

Bakhshi B, Mohseni Fard E, Nikpay N, Ebrahimi MA, Bihamta MR, Mardi M, et al. MicroRNA signatures of drought signaling in rice root. PLoS One. 2016;11: e0156814.

Baldrich P, Campo S, Wu MT, Liu TT, Hsing YI, San SB. MicroRNA-mediated regulation of gene expression in the response of rice plants to fungal elicitors. RNA Biol. 2015:12:847-63.

Baldrich P, Hsing Y, San SB. Genome-wide analysis of polycistronic microRNAs in cultivated and wild rice. Genome Biol Evol. 2016:8:1104-14.

Baldrich P, San SB. MicroRNAs in rice innate immunity. Rice. 2016;9:6.
Bialas A, Zess EK, De la Concepcion JC, Franceschetti M, Pennington HG, Yoshida $\mathrm{K}$, et al. Lessons in effector and NLR biology of plant-microbe systems. Mol Plant-Microbe Interact. 2018;31:34-45.

Boccara M, Sarazin A, Thiebeauld O, Jay F, Voinnet O, Navarro L, et al. The Arabidopsis miR472-RDR6 silencing pathway modulates PAMP- and effectortriggered immunity through the post-transcriptional control of disease resistance genes. PLoS Pathog. 2014;10:e1003883.

Campo S, Peris-Peris C, Sire C, Moreno AB, Donaire L, Zytnicki M, et al. Identification of a novel microRNA (miRNA) from rice that targets an alternatively spliced transcript of the Nramp6 (Natural resistance-associated macrophage protein 6) gene involved in pathogen resistance. New Phytol. 2013;199:212-27.

Canto-Pastor A, Santos B, Valli A, Summers W, Schornack S, Baulcombe D. Enhanced resistance to bacterial and oomycete pathogens by short tandem target mimic RNAs in tomato. Proc Natl Acad Sci U S A. 2019;116:2755-60.

Chandran V, Wang H, Gao F, Cao X-L, Chen Y-P, Li G-B, et al. miR396-OsGRFs module balances growth and rice blast disease-resistance. Front. Plant Sci. 2019:9:1999.

Dai X, Zhuang Z, Zhao P. psRNATarget: a plant small RNA target analysis server (2017 release). Nucleic Acids Res. 2018;46:W49-54.

Deng Y, Zhai K, Xie Z, Yang D, Zhu X, Liu J, et al. Epigenetic regulation of antagonistic receptors confers rice blast resistance with yield balance. Science. 2017;355:962-5.

Dong S, Zhang J, Sun D, Liu H, Yang Q, Wang H, et al. Identification of Magnaporthe oryzae-elicited rice novel miRNAs and their targets by miRNA and degradome sequencing. Eur J Plant Pathol. 2018;151:629-47.

Duan P, Ni S, Wang J, Zhang B, Xu R, Wang Y, et al. Regulation of OsGRF4 by OsmiR396 controls grain size and yield in rice. Nat Plants. 2015;2:15203.

Fang Y, Xie K, Xiong L. Conserved miR164-targeted NAC genes negatively regulate drought resistance in rice. J Exp Bot. 2014;65:2119-35.

Gao F, Wang K, Liu Y, Chen Y, Chen P, Shi Z, et al. Blocking miR396 increases rice yield by shaping inflorescence architecture. Nat Plants. 2015;2:15196.

German MA, Pillay M, Jeong D-H, Hetawal A, Luo S, Janardhanan P, et al. Global identification of microRNA-target RNA pairs by parallel analysis of RNA ends. Nat Biotechnol. 2008;26:941-6.

Huang J, Li Z, Zhao D. Deregulation of the OsmiR160 target gene OsARF18 causes growth and developmental defects with an alteration of auxin signaling in rice. Sci Rep. 2016a;6:29938.

Huang J, Yang M, Zhang X. The function of small RNAs in plant biotic stress response. J Integr Plant Biol. 2016b;58:312-27.

Kapoor M, Arora R, Lama T, Nijhawan A, Khurana JP, Tyagi AK, et al. Genomewide identification, organization and phylogenetic analysis of dicer-like, Argonaute and RNA-dependent RNA Polymerase gene families and their expression analysis during reproductive development and stress in rice. BMC Genomics. 2008;9:451

Li S, Gao F, Xie K, Zeng X, Cao Y, Zeng J, et al. The OsmiR396c-OsGRF4-OsGIF1 regulatory module determines grain size and yield in rice. Plant Biotechnol $\mathrm{J}$. 2016a;14:2134-46.

Li X, Lian H, Zhao Q, He Y. MicroRNA166 monitors SPOROCYTELESS/NOZZLE (SPL/NZZ) for building of the anther internal boundary. Plant Physiol. 2019b;181:208-20.

Li Y, Cao X-L, Zhu Y, Yang X-M, Zhang K-N, Xiao Z-Y, et al. Osa-miR398b boosts $\mathrm{H}_{2} \mathrm{O}_{2}$ production and rice blast disease-resistance via multiple superoxide dismutases. New Phytol. 2019a;222:1507-22.

Li Y, Lu YG, Shi Y, Wu L, Xu YJ, Huang F, et al. Multiple rice microRNAs are involved in immunity against the blast fungus Magnaporthe oryzae. Plant Physiol. 2014;164:1077-92.

Li Y, Zhang Q, Zhang J, Wu L, Qi Y, Zhou JM. Identification of microRNAs involved in pathogen-associated molecular pattern-triggered plant innate immunity. Plant Physiol. 2010a;152:2222-31.

Li Y, Zhao SL, Li JL, Hu XH, Wang H, Cao XL, et al. Osa-miR169 negatively regulates rice immunity against the blast fungus Magnaporthe oryzae. Front Plant Sci. 2017:8:2.

Li YF, Zheng Y, Addo-Quaye C, Zhang L, Saini A, Jagadeeswaran G, et al. Transcriptome-wide identification of microRNA targets in rice. Plant J. 2010b; 62:742-59.

Li Z, Peng J, Wen X, Guo H. Ethylene-insensitive3 is a senescence-associated gene that accelerates age-dependent leaf senescence by directly repressing miR164 transcription in Arabidopsis. Plant Cell. 2013;25:3311-28.

Li ZY, Xia J, Chen Z, Yu Y, Li QF, Zhang YC, et al. Large-scale rewiring of innate immunity circuitry and microRNA regulation during initial rice blast infection. Sci Rep. 2016b;6:25493. 
Liu B, Li JF, Ao Y, Qu J, Li Z, Su J, et al. Lysin motif-containing proteins LYP4 and LYP6 play dual roles in peptidoglycan and chitin perception in rice innate immunity. Plant Cell. 2012;24:3406-19.

Liu B, Li P, Li X, Liu C, Cao S, Chu C, et al. Loss of function of OsDCL1 affects microRNA accumulation and causes developmental defects in rice. Plant Physiol. 2005;139:296-305.

Mentlak TA, Kombrink A, Shinya T, Ryder LS, Otomo I, Saitoh H, et al. Effectormediated suppression of chitin-triggered immunity by Magnaporthe oryzae is necessary for rice blast disease. Plant Cell. 2012;24:322-35.

Navarro L, Dunoyer P, Jay F, Arnold B, Dharmasiri N, Estelle M, et al. A plant miRNA contributes to antibacterial resistance by repressing auxin signaling. Science. 2006;312:436-9.

Navarro L, Jay F, Nomura K, He SY, Voinnet O. Suppression of the microRNA pathway by bacterial effector proteins. Science. 2008;321:964-7.

Park C, Shirsekar G, Bellizzi M, Chen S, Songkumarn P, Xie X, et al. The E3 ligase APIP10 connects the effector AvrPiz-t to the NLR receptor Piz-t in rice. PLoS Pathog. 2016;12:e1005529.

Peris-Peris C, Serra-Cardona A, Sánchez-Sanuy F, Campo S, Ariño J, Segundo BS. Two NRAMP6 isoforms function as iron and manganese transporters and contribute to disease resistance in rice. Mol Plant-Microbe Interact. 2017;30: 385-98.

Quoc NB, Phuong NDN, Trang HTT, Phi NB, Chau NNB. Expression of osa-miR7695 against the blast fungus Magnaporthe oryzae in Vietnamese rice cultivars. Eur J Plant Pathol. 2019;155:307-17.

Rogers K, Chen X. Biogenesis, turnover, and mode of action of plant microRNAs. Plant Cell. 2013;25:2383-99.

Salvador-Guirao R, Baldrich P, Tomiyama S, Hsing Y, Okada K, Segundo BS. OsDCL 1 a activation impairs phytoalexin biosynthesis and compromises disease resistance in rice. Ann Bot. 2019;123:79-93.

Salvador-Guirao R, Hsing Yl, San SB. The polycistronic miR166k-166h positively regulates rice immunity via post-transcriptional control of EIN2. Front Plant Sci. 2018;9:337.

Seo JK, Wu J, Lii Y, Li Y, Jin H. Contribution of small RNA pathway components in plant immunity. Mol Plant-Microbe Interact. 2013;26:617-25.

Shimizu T, Nakano T, Takamizawa D, Desaki Y, Ishii-Minami N, Nishizawa Y, et al. Two LysM receptor molecules, CEBiP and OsCERK1, cooperatively regulate chitin elicitor signaling in rice. Plant J. 2010;64:204-14.

Shivaprasad PV, Chen HM, Patel K, Bond DM, Santos BA, Baulcombe DC. A microRNA superfamily regulates nucleotide binding site-leucine-rich repeats and other mRNAs. Plant Cell. 2012;24:859-74.

Song X, Li Y, Cao X, Qi Y. MicroRNAs and their regulatory roles in plantenvironment interactions. Annu Rev Plant Biol. 2019:70:489-525.

Tang J, Chu C. MicroRNAs in crop improvement: fine-tuners for complex traits. Nat Plants. 2017;3:17077.

Tripathi A, Chacon O, Singla-Pareek SL, Sopory SK, Sanan-Mishra N. Mapping the microRNA expression profiles in glyoxalase over-expressing salinity tolerant rice. Curr Genomics. 2018;19:21-35.

Wang B-H, Ebbole DJ, Wang Z-H. The arms race between Magnaporthe oryzae and rice: diversity and interaction of Avr and R genes. J Integr Agric. 2017;16: 2746-60.

Wang $H$, Jiao $X$, Kong $X$, Hamera S, Wu Y, Chen X, et al. A signaling cascade from miR444 to RDR1 in rice antiviral RNA silencing pathway. Plant Physiol. 2016; 170:2365-77.

Wang J, Zhou L, Shi H, Chern M, Yu H, Yi H, et al. A single transcription factor promotes both yield and immunity in rice. Science. 2018b;361:1026-8.

Wang Z, Xia Y, Lin S, Wang Y, Guo B, Song X, et al. Osa-miR164a targets OsNAC60 and negatively regulates rice immunity against the blast fungus Magnaporthe oryzae. Plant J. 2018a;95:584-97.

Wei L, Gu L, Song X, Cui X, Lu Z, Zhou M, et al. Dicer-like 3 produces transposable element-associated 24-nt siRNAs that control agricultural traits in rice. Proc Natl Acad Sci U S A. 2014;111:3877-82.

Wu H, Ma Y, Chen T, Wang M, Wang X. PsRobot: a web-based plant small RNA meta-analysis toolbox. Nucleic Acids Res. 2012;40:W22-8.

Wu J, Yang R, Yang Z, Yao S, Zhao S, Wang Y, et al. ROS accumulation and antiviral defence control by microRNA528 in rice. Nat Plants. 2017; 3:16203.

Wu J, Yang Z, Wang Y, Zheng L, Ye R, Ji Y, et al. Viral-inducible Argonaute18 confers broad-spectrum virus resistance in rice by sequestering a host microRNA. eLife. 2015;4:e05733.

Wu L, Zhang Q, Zhou H, Ni F, Wu X, Qi Y. Rice microRNA effector complexes and targets. Plant Cell. 2009;21:3421-35.
Wu L, Zhou H, Zhang Q, Zhang J, Ni F, Liu C, et al. DNA methylation mediated by a microRNA pathway. Mol Cell. 2010;38:465-75.

Xiao Z-Y, Wang Q-X, Wang H, Li J-L, Zhao S-L, Fan J, et al. MiR444b.2 regulates resistance to Magnaporthe oryzae and tillering in rice. Acta Phytopathola Sin. 2017;47:511-22 (in Chinese). http://zwblxb.magtech.com.cn/CN/10.13926/j. cnki.apps.000019.

Xie M, Zhang S, Yu B. MicroRNA biogenesis, degradation and activity in plants. Cell Mol Life Sci. 2015;72:87-99.

Xie Z, Yan B, Shou J, Tang J, Wang X, Zhai K, et al. A nucleotide-binding siteleucine-rich repeat receptor pair confers broad-spectrum disease resistance through physical association in rice. Philos Trans R Soc B. 2019;374:20180308.

Xu W, Meng Y, Wise RP. Mla- and Rom1-mediated control of microRNA398 and chloroplast copper/zinc superoxide dismutase regulates cell death in response to the barley powdery mildew fungus. New Phytol. 2014;201:1396-412.

Yao S, Yang Z, Yang R, Huang Y, Guo G, Kong X, et al. Transcriptional regulation of miR528 by OsSPL9 orchestrates antiviral response in rice. Mol Plant. 2019; 12:1114-22.

Yan Y, Wang H, Hamera S, Chen X, Fang R. MiR444a has multiple functions in the rice nitrate-signaling pathway. Plant J. 2014;78:44-55.

Yang C, Li W, Cao J, Meng F, Yu Y, Huang J, et al. Activation of ethylene signaling pathways enhances disease resistance by regulating ROS and phytoalexin production in rice. Plant J. 2017:89:338-53.

Zhai J, Jeong D-H, De Paoli E, Park S, Rosen BD, Li Y, et al. MicroRNAs as master regulators of the plant $N B-L R R$ defense gene family via the production of phased, trans-acting siRNAs. Genes Dev. 2011;25:2540-53.

Zhang D, Liu M, Tang M, Dong B, Wu D, Zhang Z, et al. Repression of microRNA biogenesis by silencing of OSDCL1 activates the basal resistance to Magnaporthe oryzae in rice. Plant Sci. 2015;237:24-32

Zhang X, Bao Y, Shan D, Wang Z, Song X, Wang J, et al. Magnaporthe oryzae induces the expression of a microRNA to suppress the immune response in rice. Plant Physiol. 2018;177:352-68.

Zhang X, Zhao H, Gao S, Wang W, Katiyar-Agarwal S, Huang H, et al. Arabidopsis Argonaute 2 regulates innate immunity via miRNA393(*)-mediated silencing of a Golgi-localized SNARE gene, MEMB12. Mol Cell. 2011;42:356-66.

Zhao ZX, Feng Q, Cao XL, Zhu Y, Wang H, Chandran V, et al. Osa-miR167d facilitates infection of Magnaporthe oryzae in rice. J Integr Plant Biol. 2019. https://doi.org/10.1111/jipb.12816.

Zhu H, Hu F, Wang R, Zhou X, Sze SH, Liou LW, et al. Arabidopsis Argonaute10 specifically sequesters miR166/165 to regulate shoot apical meristem development. Cell. 2011;145:242-56.

\section{Ready to submit your research? Choose BMC and benefit from:}

- fast, convenient online submission

- thorough peer review by experienced researchers in your field

- rapid publication on acceptance

- support for research data, including large and complex data types

- gold Open Access which fosters wider collaboration and increased citations

- maximum visibility for your research: over $100 \mathrm{M}$ website views per year

At $\mathrm{BMC}$, research is always in progress.

Learn more biomedcentral.com/submissions 